Historic, Archive Document

Do not assume content reflects current scientific knowledge, policies, or practices. 



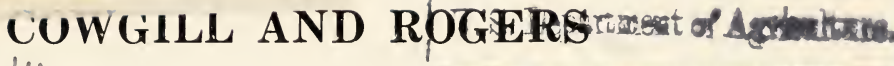

"On both sides of the Bay"

\section{PERENNIALS, ALPINES AND ROCK PLANTS}

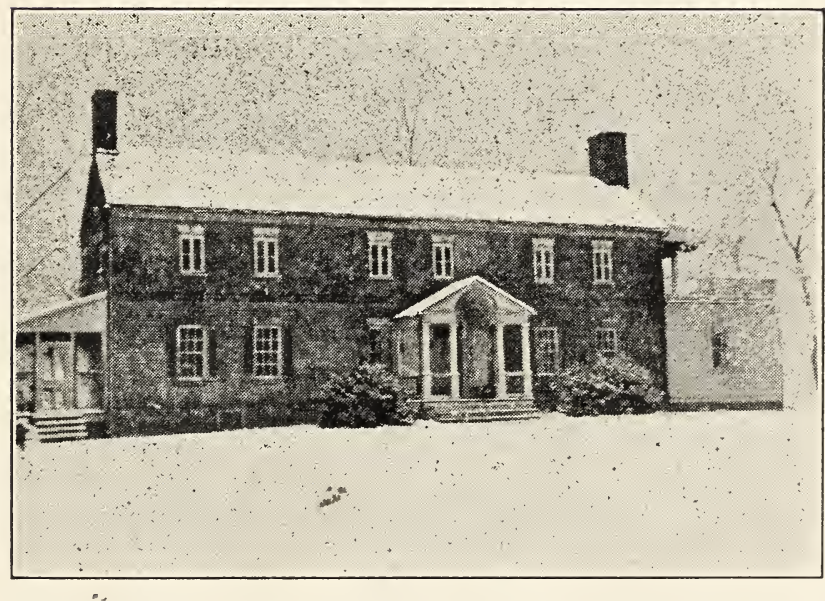

Belvoir Manor where Francis Scott Key spent part of his childhood and youth. Built about 1730 by his great grandfather.

MISS KATHLEEN COWGILL

THE LOMBARDY NURSERIES EASTON, MD.
MRS. WM, COLEMAN ROGERS BELVOIR MANOR FLOWER FARM WATERBURY, A. A. CO., MD. 



\section{More Than a Commercial Aim}

For a number of years Miss Kathleen Cowgill and Mrs. William Coleman Rogers, proprietors of the combined Lombardy Nurseries and Belvoir Manor Farms, devoted themselves to plant collecting as a hobby.

They made more or less of a specialty of rare specimens, alpines and perennials from various countries and of experimenting with them, with the object of learning which of them were adaptable to Maryland soil and climatic conditions.

Their work and observation, so painstakingly and lovingly followed, put them in the way of many delightful discoveries and resulted further in the accumulation of a fund of knowledge about different species and varieties decidedly worth handing on to others interested in the more unusual plant material.

Hence, when they finally decided to enter the professional growers' field, they did so, not only with the assurance which experience and thorough information alone can provide, but with the desire to meet exacting demands and, at the same time, to establish such a relationship with their patrons as exists, say, between the dealer in fine objects of art or books with his clientele.

The Lombardy-Belvoir Manor establishment, in a word, has far more than a merely commercial aim. It hopes to be the means of iritroducing into Maryland gardens many beautiful plants which their owners, perhaps, do not dream can be grown in this state.

Among these flowers grown from seed imported from England and other foreign lands, rock plants and other perennials that will bring an unaccustomed but very decidedly charming note of novelty to any collection.

The growing fields are located almost exactly opposite each other with the Chesapeake Bay between-the Lombardy Nurseries at Easton and the Belvoir Manor Flower Farms at Waterbury, Anne Arundel County. This difference of exposure and soil has given these experiments a broader scope than would otherwise have been possible. When you buy this stock, you may be assured that it is adaptable to the requirements of this part of the country.

Because the proprietors have a special flair for the unusual in plant life, does not mean that they pay no attention to the commoner things. Far from it. For this collection includes most of the dependable standbys without which no garden is complete.

In addition to filling nursery orders, Miss Cowgill and Mrs. Rogers maintain a Landscaping Department equipped to carry out any kind of a plan desired or to supply original ideas. In the designs they suggest, the aim is to get away from the exotic garden which so often is entirely out of place in any but the locality of which it is characteristic. Emphasis is put upon schemes that really "belong"-that are, so to speak-part and parcel of Maryland tradition and atmosphere. 


\section{Terms}

All shipments are sent at purchaser's expense, by express, parcel post, or freight.

All plants are sold and all plans and plantings are made for cash only.

Plants, except in the case of a few which can be transplanted from pots only, are all large field grown specimens, which will bloom the first year if planted early. There are a few, such as peonies, which bloom only after becoming thoroughly established.

Plants are sent out in good condition. We are not responsible for them after they leave our hands. Colors of plants grown from seed cannot be guaranteed. All plants grown from division or cuttings will be replaced if not true to name and color.

Prices in this catalogue are subject at all times to changes in the market. All are sold subject to above conditions.

We maintain a landscape service and will gladly submit plans or give advice and cultural directions. We quote roses, shrubs and evergreens if desired. Our box specimens are especially fine.

Members of the firm are open to engagements to speak before clubs on garden subjects.

Direction for reaching Belvoir Manor: cross Hanover Street Bridge, through Glen Burnie on South Shore Boulevard, through Cross Roads and Crownsville.

Phone W. C. Rogers, Annapolis 1857-F-12.

Additions to this Catalogue will be sent out from time to time.

VISITORS ARE WELCOMED AT ALL TIMES.

Visitors may select blooming plants to be delivered in planting season. 


\section{COWGILL \& ROGERS}

\section{UNUSUAL FOREIGN, ENGLISH, AND AMERICAN PERENNIALS, ALPINES, AND ROCK PLANTS}

Box Specimens or Edging, Shrubs, Roses, and Evergreens. $(1 / 2$ dozen at dozen rate)

Eastern Shore Fruit Cake $\$ 1.00$ the lb.
Belvoir "Vintage" Hams 1 yr. $70 \mathrm{c}, 2$ yr. $80 \mathrm{c}$ the $\mathrm{lb}$.

\section{Section 1.-Alpines and Rock Plants}

Each Doz.

1 Adonis amurensis, yellow, very early. $\$ .50$

2 Aethionema pulchellum, lavender, spring...................... $\quad \mathbf{. 3 0}$

3 Alyssum saxatile compactum, yellow, spring............... $\quad .25$

4 Anchusa myosotidiflora, lovely blue, spring................. $\quad . \mathbf{4 0}$

5 Androsace brigantiaca, white, spring............................... $\quad \mathbf{4 0}$

6 Androsace laggeri, deep rose, spring................................ $\quad .40$

7 Androsace sempervivoides, purple, spring...................... $\quad .40$

8 Anemone St. Brigid, vivid colors, spring........................ $\quad .25$

9 Aquilegia alpina, blue, spring............................................ $\quad .25$

10 Aquilegia flabellata, white, spring.................................... $\quad .25$

11 Armeria caespitosa, pink, spring...................................... $\quad \mathbf{. 5 0}$

12 Armeria Laucheana, pink, June............................................ $\quad .25$

13 Aster alpinus, mixed, summer......................................... $\quad .25$

14 Aster Mauve Cushion, November.................................... $\quad .25$

15 Aubrietia Hybrids, lovely colors, spring.......................... $\quad .25$ $\$ 2.50$

16 Bellis perennis (English Daisy), pink and white, very early ......................................................................... .15

17 Campanula carpatica (hairbell), blue summer.............

18 Campanula Laurii, lovely blue, summer.......................... $\quad . \mathbf{3 0}$

19 Campanula Robsoni, fine blue, summer........................... $\quad \mathbf{3 0}$

20 Campanula velutina, pink, magnificent............................ $\quad . \mathbf{4 0}$

21 Cerastium Biebersteinii, white, summer.......................... $\quad .25$

22 Cerastium tomentosum, white, summer.......................... $\quad .25$

23 Cheiranthus Allionii, orange, perpetual........................... $\quad .20$

24 Cheiranthus linofolius, lavender, winter and fall........ $\quad .25$

25 Cheiranthus (Wallflower), mixed, spring. 
Each Doz.

26 Delphinium chinense, Blue Butterfly............................ .25

27 Dianthus caesius, pink.

28 Dodecatheon radicatum, pink........................................... $\quad .30$

29 Erigeron caucasicus, lavender, summer......................... $\quad .25$

30 Erigeron multiradiatus roseus, summer......................... $\quad .25$

31 Erigeron mucronatus, lavender, summer.......................... $\quad .25$

32 Gentiana acaulis, blue, summer........................................ $\quad .50$

33 Gentiana biglovei, blue, summer................................... $\quad .50$

34 Gentiana cruciata, blue, summer...................................... $\quad .50$

35 Gentiana Parryi, blue, summer........................................... $\quad .50$

36 Gentiana thibetica, yellow, summer.................................. $\quad .50$

37 Gypsophila repens, white, spring........................................ $\quad .25$

38 Gypsophila repens rosea, spring................................... .25

39 Helianthemum grandiflorum, mixed, all summer........ $\quad .25$

40 Helleborus niger (Christmas Rose), white, winter... 1.00

41 Helleborus orientalis, mixed hybrids, winter................. 1.00

42 Heuchera hybrids, brilliant colors, summer................. $\quad .25$

43 Hieracium aurantiacum, orange, summer....................... .25

44 Horminum pyrenaicum, violet........................................... $\quad .30$

45 Hypericum repens, gold, summer.................................... .25

46 Iberis gibraltarica, lavender, spring................................ .25

47 Iberis sempervirens, white, spring.................................... $\quad .25$

48 Iris cristata, lavender, spring.............................................. .25

49 Lithospermum, yellow............................................................30

50 Maivastrum coccineum, copper scarlet......................... $\quad .30$

51 Myosotis alpestris, blue, spring........................................ .15

52 Myosotis alpestris, Triumph, blue, spring...................... .15

53 Myosotis dissitiflora, blue, spring..................................... .15

54 Myosotis palustris grandiflorum, blue, summer........ .15

55 Penstemon Scouleri, lilac, shrubby................................... $\quad .30$

56 Phlox amoena, rosea, rose, spring................................... $\quad .25$

57 Phlox divaricata, lavender, spring................................ $\quad .25$

58 Phlox divaricata Laphami, blue, spring........................ .25

59 Phlox multiflora, pink, spring............................................. .25

60 Phlox subulata G. F. Wilson, lilac, spring ...................... .25

61 Phlox subulata, white, spring.

62 Primula Bartley Strain, direct from Dalrymple the originator

.25

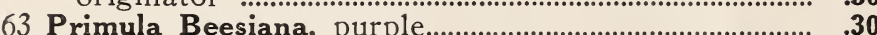

64 Primula Bulleyana, red.......................................................... $\quad .30$

65 Primula denticulata, lavender.............................................. $\quad . \mathbf{3 0}$

66 Primula denticulata alba.................................................. $\quad .30$

67 Primula denticulata cashmeriana, lavender, dwarf.... $\quad .30$ 
Each Doz.

68 Primula chionantha, white............................................... $\quad \mathbf{3 0}$

69 Primula japonica Etna, apricot......................................... $\quad .30$

70 Primula polyanthus, Ipswich strain................................. $\quad . \mathbf{3 0}$

71 Primula polyanthus, mixed.................................................. $\quad .25$

72 Primula polyanthus, Munstead White............................. $\quad .30$

73 Primula pulverulenta, purple............................................ $\quad . \quad 30$

74 Primula pulverulenta, Annesgrove Hybrid, pink...... $\quad .30$

75 Primula veris, mixed............................................................... $\quad .25$

76 Primula veris, Ruby Red..................................................... $\quad .25$

77 Primula veris, Spetchley strain........................................... $\quad .30$

78 Primula veris, yellow.............................................................. $\quad .25$

79 Saxifraga Kolenatiana, pink .................................................. $\quad .40$

80 Saxifraga muscoeides atropurpurea, red........................ $\quad . \mathbf{4 0}$

81 Sedum acre, yellow, evergreen, good hanging vine.. $\quad .25$

82 Sedum maximum, white, fall................................................. $\quad .25$

83 Sedum spectabile, pink, fall.............................................. $\quad .25$

84 Sedum Sieboldii, pink, fall................................................ $\quad .25$

85 Sempervivum (Houseleeks) ................................................ $\quad .30$

86 Stachys alpina.................................................................... $\quad .25$

87 Tunica saxifraga, pink, summer....................................... $\quad .25$

88 Valeriana acutiloba, pink and white............................... $\quad . \mathbf{3 0}$

89 Verbena venosa, lavender, summer................................ $\quad .25$

90 Veronica corymbosa stricta, lovely blue.......................... $\quad .25$

91 Veronica Guthriana, good blue.......................................... $\quad .25$

92 Veronica prostrata, brilliant blue, carpeter................ $\quad .25$

93 Veronica repens, bright blue, carpeter.......................... $\quad .25$

94 Veronica Royal Blue, dwarf bush................................... . .25

95 Veronica rupestris, fine blue, carpeter........................... .25

96 Viola cornuta, Jersey Gem, violet.................................... $\quad .30$

97 Viola cornuta, mixed............................................................. $\quad .25$

98 Viola native species, violet blue .......................................... $\quad .25$

99 Viola native species, white marked blue........................... .25

100 Wahlenbergia dalmatica, deep violet.................................. $\mathbf{3 0}$ 


\section{Section 2.-Perennials for Borders}

\section{LARGE FIELD GROWN PLANTS}

Each Doz.

101 Achillea filipendulina, butter yellow, $3 \mathrm{ft}$., June and July

102 Achillea millefolium roseum, $11 / 2$ ft., June and July..

103 Achillea ptarmica, The Pearl, white, 2 ft., summer....

104 Aconitum Fischeri, blue, 2 ft., September.....................

105 Aconitum napellus, blue, $3 \mathrm{ft}$., July.

106 Aconitum Wilsoni, blue, $6 \mathrm{ft}$., October.

107 Anemone hupehensis, lilac, $2 \mathrm{ft}$., August.

108 Anemone japonica, Queen Charlotte, pink, 3 ft., September and October.

109 Anemone japonica, Whirlwind, double white, $3 \mathrm{ft}$., September and October.

110 Anchusa Barrelieri, blue, 11/2 ft., April and July........

111 Anchusa italica Dropmore, blue, $4 \mathrm{ft}$., May and June

112 Anchusa italica Lissadell, blue, $4 \mathrm{ft}$., May and June..

113 Anchusa italica Opal, blue, $4 \mathrm{ft}$., May and June........

114 Aquilegia (Columbine) Barr's Pinks, $3 \mathrm{ft}$., May and June

115 Aquilegia chrysantha, clear yellow, 3 ft., June and July

116 Aquilegia Helenae, blue and white, 2 ft., April and May

117 Aquilegia Mrs. Scott Elliott's mixed, long spurred, 3 ft., May and June.

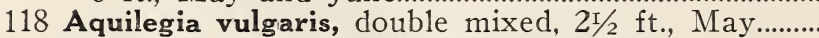

119 Aquilegia vulgaris, Munstead White, $2 \mathrm{~T} / 2 \mathrm{ft}$., April and May

120 Artemisia lactiflora, 3 ft., pearl-like blooms in August

121 Asclepias tuberosa (Butterfly Plant), orange, $2 \mathrm{ft}$., July and August

122 Aster (hardy) amellus hybrids, lovely shades, $2 \mathrm{ft}$., July on

123 Aster Barr's Pink, 4 ft., July on.

124 Aster Blue Boy, $3 \mathrm{ft}$., July on.

125 Aster Climax, blue, September

129 Aster novae angliae, purple, $5 \mathrm{ft}$., August on.............

127 Aster King of the Belgians, blue, October. .25 
Each Doz.

129 Aster novae angliae, mixed, purple, $5 \mathrm{ft}$., August on

130 Aster novae angliae, rose, $5 \mathrm{ft}$., September................

131 Aster St. Egwin, shell pink, very lovely, 2 ft., September and October.

132 Aster Tartaricus, lavender, $5 \mathrm{ft}$., Oct. and Nov..

133 Bocconia cordata (Plume Poppy), creamy plumes, $5 \mathrm{ft} .$, June and July...

134 Boltonia asteroides, white, $4 \mathrm{ft}$., August, September

135 Boltonia latisquama, pink, 4 ft., Aug., Sept................

136 Buddleia magnifica, lavender, 5 ft., all summer, shrub

137 Campanula alliariaefolia (bell flower), cream, $3 \mathrm{ft}$., June

138 Campanula medium (Canterbury Bells), mixed...........

139 Campanula celtidifolia, blue, $2 \mathrm{r} / 2 \mathrm{ft}$, summer................

140 Campanula celtidifolia alba magnifica, white, $5 \mathrm{ft}$., summer

141 Campanula celtidifolia Edw. Molyneux, blue, summer, extra good.

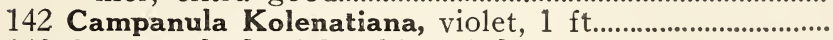

143 Campanula latifolia, blue, $2 \mathrm{ft}$.

144 Campanula persicifolia (peach bells), English hybrids, lovely, $3 \mathrm{ft}$., June......

145 Caryopteris mastacanthus (Blue Spirea), 2 ft., blue, September, shrubby.........................................................

146 Centaurea montana (Perennial Cornflower), blue, summer, 2 ft.

147 Chelone lyoni (Shellflower), pink, 2 ft., August...........

148 Chrysanthemum, button bronze.........................................

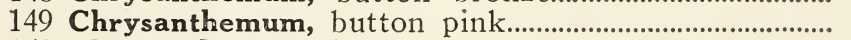

150 Chrysanthemum, button yellow..............................................

151 Chrysanthemum, large pinks...............................................

152 Chrysanthemum, large red....................................................

153 Chrysanthemum, large white................................................

154 Chrysanthemum, large yellow.........................................

155 Clematis integrifolia, blue, bushy, August.......................

156 Coreopsis lanceolata grandiflora, gold, 3 ft., all summer ............................................................................ .25

157 Cynoglossum Wallichii, blue, all summer..

158 Delphinium belladonna, pale blue, May on.....................

159 Delphinium from Blackmore \& Langdon's, mixed sorts

160 Delphinium Gold Medal strain. 
Each Doz.

162 Delphinium Vanderbilt Hybrids..................................... $.25 \quad 2.50$

163 Dianthus barbatus, crimson............................................... $\quad .20 \quad 2.00$

164 Dianthus barbatus, mixed................................................... $\quad .20 \quad 2.00$

165 Dianthus barbatus, Newport Pink................................... $\quad .20 \quad 2.00$

166 Dianthus barbatus, salmon scarlet.................................. $\quad .20 \quad \mathbf{2 0} \quad \mathbf{2 . 0 0}$

167 Dianthus barbatus, white.................................................... $\quad .20 \quad 2.00$

168 Dianthus plumarius (clove pinks), mixed..................... $\quad .25 \quad 2.50$

169 Digitalis (Foxglove) ambigua, yellow, 2 ft., May (perennial)

$.25 \quad 2.50$

170 Digitalis ferruginea, cream, lavender dots, $4 \mathrm{ft.}$, July (perennial) ................................................................ .25

2.50

171 Digitalis laevigata, yellow, $2 \mathrm{ft} .$, May (perennial)....

172 Digitalis lutea, yellow, very dainty, $2 \mathrm{ft}$., May, June

173 Digitalis Lutz hybrids, salmon shades, $5 \mathrm{ft}$., May......

174 Digitalis ochroleuca, yellow, 2 ft., May..........................

175 Digitalis Shirley, mixed, $5 \mathrm{ft}$

.25

2.50

$.25 \quad 2.50$

$.25 \quad 2.50$

$.25 \quad 2.50$

$.25 \quad 2.50$

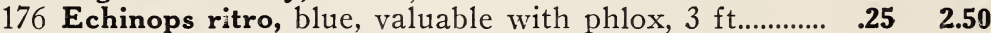

177 Eryngium amethystinum, blue, summer, $2 \mathrm{ft}$., good with phlox

2.50

178 Euonymus radicans, rare orange berried shrub........

179 Eupatorium coelestinum, blue, $1 \mathrm{1} / 2 \mathrm{ft}$

.30

.25

2.50

180 Funkia lanceolata (Plaintain Lily), lavender, $1 \mathrm{ft}$., September

.25

2.50

181 Funkia coerulea, lavender purple, 2 ft., July.............. .25

2.50

182 Funkia sub-cordata grandiflora, white, 2 ft., August

183 Gaillardia, English hybrids, mixed, $2 \mathrm{ft}$., all summer

184 Galega Vilmorin, sweet pea-like blossoms of lavender, all summer, $2 \mathrm{ft}$.

.25

2.53

.25

2.50

.25

2.50

185 Geum astrosanguieum, red, $1 \mathrm{ft}$., summer...................... .25

2.50

186 Gypsophila acutifolia, white, $2 \mathrm{ft}$., July

.25

.25

2.50

187 Gypsophila paniculata, white, 2 ft., summer.................

188 Helenium autumnale superbum, yellow, $5 \mathrm{ft}$., August and September

.25

2.50

189 Helenium Riverton Gem, wallflower red, changing to old gold, $5 \mathrm{ft} .$, August and September.

.25

2.50

2.50

2.50

191 Heliopsis, golden yellow, June on, $4 \mathrm{ft}$........................... .25

2.50

192 Hemerocallis flava (lemon lily), yellow, $3 \mathrm{ft}$., May....

193 Hemerocallis Florham, yellow, $3 \mathrm{ft}$., June......................

194 Hemerocallis fulva, copper, $3 \mathrm{ft}$., July.

$\begin{array}{ll}.25 & 2.50 \\ .25 & 2.50\end{array}$

195 Hemerocallis Thunbergii, waxy yellow, $4 \mathrm{ft}$., July and August 
Each Doz.

196 Hesperis matronalis (Sweet Rocket), shades of lavender and white, $3 \mathrm{ft}$. , May................................... $\quad .25$

197 Hibiscus Golden Bowl, translucent, yellow cups with garnet eyes, $6 \mathrm{ft}$................................................ .25

198 Hibiscus giant flowering, pink and white, 4 ft., July

199 Hibiscus Mallow Marvels, crimson, pink and white, 4 ft., July

200 Hollyhock, Exquisite, mixed, fringed ruffled, double

201 Hollyhock, double, crimson, Newport Pink, white, yellow or salmon.

202 Hollyhock, Imperator, large, like a single Japanese peony with a fringed center, mixed (new).............. $\quad . \mathbf{3 0}$

203 Hollyhock, single mixed................................................... .25

204 Iris Archeveque, glowing purple.......................................... $\quad .50$

205 Iris Aurora, lavender pink................................................ $\quad .50$

206 Iris Celeste, pale blue...........................................................25

207 Iris Crusader, clear blue................................................... $\quad \mathbf{. 5 0}$

208 Iris Empire, yellow............................................................ $\quad .50$

209 Iris Lohengrin, lavender.................................................. $\quad .25$

210 Iris Mlle. Schwartz, pearly, lavender.............................. 1.00

211 Iris Mlle. Yvonne Pelletier, same.................................. 1.00

212 Iris Mother of Pearl, like its name................................. $\quad .75$

213 Iris Pallida Dalmatica, light lavender............................ $\quad .50$

214 Iris Queen of May, lavender-pink.................................. $\quad .25$

215 Iris Shekinah, pale yellow, tall........................................ $\quad .75$

216 Iris Sherwin Wright, golden yellow................................ $\quad .25$

217 Iris Virginia Moore, gold.................................................. $\quad .75$

218 Iris White Knight, pure white......................................... $\quad .50$

219 Iris Dutch mixed, orchid like........................................... $\quad .25$

220 Iris English mixed, orchid like........................................ .25

221 Iris Japanese, blue, most beautiful iris......................... $\quad .40$

222 Iris Japanese, lavender....................................................... $\quad .40$

223 Iris Japanese, purple......................................................... $\quad .40$

224 Iris Japanese, white............................................................ $\quad .40$

225 Iris orientalis, blue, slim and tall.................................... .25

226 Iris orientalis, white, same habit..................................... $\quad .25$

227 Iris siberica, blue, shorter than orientalis.................... $\quad .25$

228 Iris spanish, very small like orchids.............................. $\quad .25$

4.00

4.00

4.00

4.00

2.50

2.50

2.50

229 Isatis glaucus, yellow, blooms with iris, $3 \mathrm{ft}$., like gypsophila

230 Kniphofia hybrids, many colors............................................ $\quad .25$

231 Kniphofia Stark's hybrids, May on .................................. $\quad .25$ 
Each Doz。

233 Liatris spicata, purple, $3 \mathrm{ft}$. , August................................ . .25

234 Lilium candidum (Madonna), white, $4 \mathrm{ft}$. , June......... $\quad .35 \quad 3.50$

234 Lilium regale, white, flushed pink and yellow, $3 \mathrm{ft}$.,

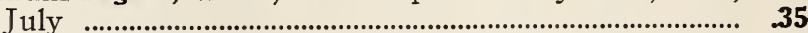

236 Lilium tigrinum (Tiger), copper, purple spots, $6 \mathrm{ft}$., July

237 Linum Lewisii, blue, $3 \mathrm{ft}$., all summer, lovely...............

238 Linum perenne, blue, 2 ft., all summer.

239 Lupinus polyphyllus, Downer's, Elliott's, and Harknesses, newest English hybrids in pink, apricot, blue, and rose, mixed.

.25

2.50

.25

2.50

.25

2.50

.25

2.50

240 Lupinus Lombardy seedlings of above, selected for hardiness

.25

2.50

241 Lythrum superbum, rose, $3 \mathrm{ft}$., July, August.................. .25

2.50

242 Malva moschata (Musk Mallow), pink, summer, $2 \mathrm{ft}$.

243 Mertensia virginica, entrancing, blue, spring flower, $2 \mathrm{ft}$.

.25

2.50

.25

2.50

244 Monarda (Bergamot), red, rose, or white, 2-3 ft., July and August.

245 Monarda hybrids, all colors mixed, very beautiful and new

.25

2.50

.30

3.00

246 Oenothera Clutei, yellow, large cups, $4 \mathrm{ft}$........................

247 Oenothera Lamarkiana, yellow, long spikes, $4 \mathrm{ft} . . . .$.

248 Oenothera speciosa, large white flowers, $2 \mathrm{ft}$...............

249 Oenothera Youngei, large orange, all summer, $2 \mathrm{ft}$.

250 Pansy, in mixed or separate colors.

251 Papaver orientalis (Oriental Poppy)

252 Papaver orientalis shades of pink, spring.

253 Papaver orientalis, red, May, June.

254 Papaver orientalis, apricot.

255 Pardanthus (Blackberry Lily), copper, $2 \mathrm{ft}$., July....

256 Penstemon barbatus, coral pink hybrids, $2 \frac{1}{2} \mathrm{ft}$.,

257 Penstemon barbatus, coral red hybrids, $2 \mathrm{r} / 2 \mathrm{ft}$., June

.25

2.50

.25

2.50

.25

.10

2.50

1.00

$.30 \quad 3.00$

.25

2.50

.30

.25

258 Penstemon heterophyllus, blue dwarf...

259 Phlox deccusata, Bridesmaid, white, pink eye.............. Large heads

.30

.25

2.50

.25

2.50

$.25 \quad 2.50$

$.25 \quad 2.50$

260 Phlox deccusata, G. H. Strohlein, salmon scarlet.......

2.50

261 Phlox deccusata, Frau Anton Buchner, white, very large

262 Phlox deccusata, Rheinlander, salmon pink, splendid

.25

2.50

.25

2.50

263 Phlox Suffruticosa, Miss Lingard, white, very early, finest white 
Each Doz.

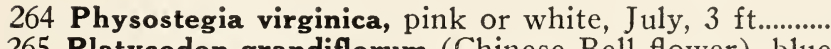
265 Platycodon grandiflorum (Chinese Bell-flower), blue or white, $2 \mathrm{ft}$., July on

266 Pyrethrum hybridum, seedlings of Kelway's named varieties, mixed

267 Rudbckia fulgida, yellow, $3 \mathrm{ft}$, all summer, large daisies

268 Rudbeckia Newmanni (perennial Black-Eyed Susan), yellow, August and September, $2 \mathrm{ft}$

269 Rudbeckia purpurea (Pink Coneflower), 3 ft., July on

270 Saponaria officinalis fl. pl. pink, July, $2 \mathrm{ft}$.

271 Salvia azurea, sky blue, $3 \mathrm{ft}$., fall.

272 Salvia farinacea, delicate blue, $3 \mathrm{ft}$., June until frost

273 Salvia glutinosa, yellow, $3 \mathrm{ft}$., summer.

.25

2.50

.25

2.50

274 Salvia nutans, violet, $2 \mathrm{ft}$

275 Salvia patens, bright blue, $2 \mathrm{ft}$., midsummer.

276 Salvia pratensis, blue, summer, $2 \mathrm{ft}$.

277 Salvia ringens, purple, $3 \mathrm{ft}$

278 Salvia sclarea, lavender, $3 \mathrm{ft}$., July splendid

279 Salvia vatican, lavender, $3 \mathrm{ft}$., very showy

280 Scabiosa caucasica, seedlings of House's, named varieties, exquisite, fall

281 Scabiosa japonica, blue, $2 \mathrm{ft}$., summer

282 Shasta Daisy, Westralia, King Edward VII and maximum, white, early, medium, and late.

283 Stokesia cyanea (Cornflower), blue or white, $2 \mathrm{ft}$., all summer

284 Thalictrum dipterocarpum, lilac, $3 \mathrm{ft}$., July and August, lovely

285 Thalictrum aquilegifolium, lavender, $3 \mathrm{ft}$., May, June 286 Tradescantia (Widow's Tears), shades of blue, May on, $1 \mathrm{I} / 2 \mathrm{ft}$

287 Trollius hybridus. (Globeflower), large globes of yellow or orange, $1 \mathrm{r} / 2 \mathrm{ft}$., May on...............................

288 Valeriana officinalis (Garden Heliotrope), exquisitely scented heads of white, $4 \mathrm{ft}$., May, June........

289 Valeriana rubra (valerian), red, pink, or white, $2 \mathrm{ft}$., all summer

.30

290 Verbascum Miss Willmott, stately spikes of ivory white, $6 \mathrm{ft}$., July on

291 Veronica amethystina, splendid blue spikes, $2 \mathrm{ft}$., all summer 
Each Doz.

292 Veronica longfolia subsessilis, one of the best; deep blue, 3 ft., August.................................................. .25

2.50

293 Veronica michauxioides, very fine, light blue, continuous bloomer, $4 \mathrm{ft}$.

294 Veronica salicifolia, lavender, shrubby.

.30

295 Veronica True Blue, erect, bright blue spikes, all summer, $2 \mathrm{ft}$.

296 Veronica Waldsteeniana, steel blue, $4 \mathrm{ft}$

297 Vitex macrophylla (spikenard), blue shrubby, all summer .75

298 Yucca filamentosa, white, 5 ft., June.

\section{Section 3.-Annuals}

299 Ageratum, Blue Perfection.

300 Ageratum, Little Blue Star, very dwarf

301 Alyssum Lilac Queen

302 Alyssum Little Dorrit, white

303 Aster elegance, single mixed

304 Calendula, The Ball, orange

305 Calendula Lemon Queen

306 Celosia plumosa, yellow.

307 Cosmos Lady Lenox, pink

308 Marigold Orange Prince

309 Marigold Lemon Queen

310 Nicotiana hybrids

311 Petunia Diener's, giant pink or purple.

312 Petunia bedding, Rosy Morn or Violet Queen

313 Scabiosa Azure Fairy and Peachblossom

314 Snapdragons, in separate colors

315 Zinnias, in separate colors

Above plants $75 \mathrm{c}$ dozen untransplanted; $\$ 1.00$ dozen transplanted. Salvia purple, salmon or red, at $25 \mathrm{c}$ each, $\$ 2.50$ per dozen. 

